\title{
Synthesis and Characterization of Highly Dispersed Au Nanoparticles Supported on $\mathrm{Z}_{\mathrm{r}} \mathrm{O}_{2}$
}

\author{
Wenjing Cui ${ }^{a}$, Menggentuya, Hongliang Wang, Xiaolei Zhang, Jinjin Lu, and \\ Wei Hou ${ }^{\text {, * }}$
}

\author{
Department of Chemical Engineering, Inner Mongolia Vocation college of Chemical Engineering, \\ Hohhot, Inner Mongolia, 010070, China. \\ azczcwj@sina.com, bnhyhw@126.com
}

Keywords: solution, Gold nanoparticles, zirconium oxide.

\begin{abstract}
This paper investigated Nano-gold catalyst prepared by solution reduction method. TEM, XRD, AAS and XPS were employed to investigate the morphology, composition, dispersity and catalytic properties of the catalyst. The results indicated that Nano-scale metallic state gold was highly dispersed on the support. Furthermore, this preparation method leads to highly active $\mathrm{Au} / \mathrm{ZrO}_{2}$ catalyst for the selective oxidation of benzyl alcohol.
\end{abstract}

\section{Introduction}

It is well known that bulk gold is stable in air and used for currency and jewelry for many years. This idea was given up since researchers discoveried the unique catalytic activity in oxidative elimination of carbon monoxide on gold particles with size on the scale of nanometers [1]. Nowadays, supported gold catalysts (Au NPs) draw widely attention because they showed extraordinary catalytic, selective, non-toxic and recyclable properties in many reactions, such as selective oxidation of alcohols, aldehydes, amines and hydrocarbons, epoxidation of propylene, hydrochlorination of ethyne, $\mathrm{C}-\mathrm{N}$ and $\mathrm{C}-\mathrm{N}$ bond formation [2,3]. In particular, selective oxidation of alcohols on Au NPs is considered as one of the most promising reactions in green chemistry because the abundant and harmless oxidant, air or molecular oxygen, was used. The oxidation of alcohols over gold catalysts under ambient conditions (atmospheric pressure and room temperature) has been demonstrated but is still limited [4].

The traditional preparation of Au catalysts include two methods: one is impregnation, the other is deposition-precipitation. The drawbacks are obvious, lower metal loading, bigger mean diameter and inhomogeneous distribution. Therefore, much progress must be made to form high dispersion, uniform size distribution catalyst.

In this study, supported Au catalyst was synthesized by the reduction of metal precursor on the support with a reducing agent in the presence of a protective agent to prevent aggregation, which is called solution reduction method. By using this synthesis method, highly dispersed Au catalyst was formed. What is more, the catalytic activity of benzyl alcohol oxidation were examined at ambient conditions.

\section{Experimental}

\subsection{Catalyst preparation.}

$\mathrm{ZrO}_{2}$ (Nano powder, Sigma-aldrich) was used as support. $\mathrm{HAuCl}_{4} \cdot 4 \mathrm{H}_{2} \mathrm{O}$ (Sino pharm Chemical Reagent Company) was used as precursor. $\mathrm{NaBH}_{4}$ (Sigma-aldrich) and L-lysine (Sigma-aldrich) were used as reducing agent and protection agent, respectively. Preparation of $3 \mathrm{wt} \% \mathrm{Au} / \mathrm{ZrO}_{2}$ catalyst is as follows.

$\mathrm{ZrO}_{2}$ powder $1.0 \mathrm{~g}$ was dispersed into $100 \mathrm{~mL}$ aqueous solution of $0.15 \mathrm{mmol} \mathrm{HAuCl}_{4}$ was then added to the mixture under vigorous stirring for $30 \mathrm{~min}$. An aqueous solution of $\mathrm{NaBH}_{4}(0.1 \mathrm{~mol} / \mathrm{L}$, $10 \mathrm{~mL}$ ) was added gradually to the suspension in about $10 \mathrm{~min}$, followed by hydrochloric acid $(0.1$ 
$\mathrm{mol} / \mathrm{L}, 10 \mathrm{~mL}$ ). The mixture was left to stand for $24 \mathrm{~h}$ and then the solid was separated, washed with water (4 times) and ethanol (twice), and dried at $80{ }^{\circ} \mathrm{C}$.

\subsection{Catalyst characterization.}

TEM images were recorded with a Jeol JEM-1210 transmission electron microscope employing an accelerating voltage of $200 \mathrm{kV}$. The samples were suspended in ethanol and dried on holey carbon-coated $\mathrm{Cu}$ grids. The composition of some samples was determined by using the energy-dispersive X-ray spectroscopy attachment of the transmission electron microscope. Powder X-ray diffraction (XRD) patterns were recorded on Philips PW1830 diffractometer using filtered $\mathrm{Cu}-\mathrm{K} \alpha$ radiation $(\lambda=0.15405 \mathrm{~nm})$; the instrument was operated at $40 \mathrm{kV}$ and $40 \mathrm{~mA}$. Diffraction data were collected between 10.0 and $80.0^{\circ}$ with a resolution of $0.02^{\circ}(2 \theta)$ for high angles. Au loading of catalyst was determined by using atomic absorption spectrometry (AAS) on Z-8000 spectra (Japan); gold particles in the samples were dissolved with nitro hydrochloric acid. The X-ray photoelectron spectroscopy (XPS) was measured with ESCALAB210 of British VG Company. All binding energies were referenced to the $\mathrm{C}(1 \mathrm{~s})$ hydrocarbon peak at $285.00 \mathrm{eV}$.

\subsection{Catalytic experiment.}

The reactions of selective oxidation of benzyl alcohol were carried out in a stirred $25 \mathrm{ml}$ round-bottomed flask under $\mathrm{O}_{2}$ atmosphere. $50 \mathrm{mg}$ catalyst, benzyl alcohol (3 mmol), ethanol $(10 \mathrm{~mL})$ and $\mathrm{NaOH}(0.5 \mathrm{mmol})$ were charged into the flask. The suspension was stirred regularly for 24 hours at $30{ }^{\circ} \mathrm{C}$ and $40{ }^{\circ} \mathrm{C}$. During the reactions $0.1 \mathrm{ml}$ samples were periodically collected, filtered and analyzed by GC (Shimadzu GC-2014) with a capillary column of Rtx-5, temperature of column ranges from 100 to $220^{\circ} \mathrm{C}\left(6^{\circ} \mathrm{C} / \mathrm{min}\right)$, injector temperature $260{ }^{\circ} \mathrm{C}$ and flame ionization detector. The products were identified by comparison with known standard samples.

\section{Results and discussion}

\subsection{Characterization of catalysts.}

The particle size and morphology of $3 \mathrm{wt} \% \mathrm{Au} / \mathrm{ZrO}_{2}$ can be observed by transmission electron microscopy. (Figure 1) As it can be seen, the Au NPs disperse well on the $\mathrm{ZrO}_{2}$ and have mean dimensions of about $4.5 \mathrm{~nm}$. The Au content is $2.71 \mathrm{wt} \%$ according to AAS analysis.
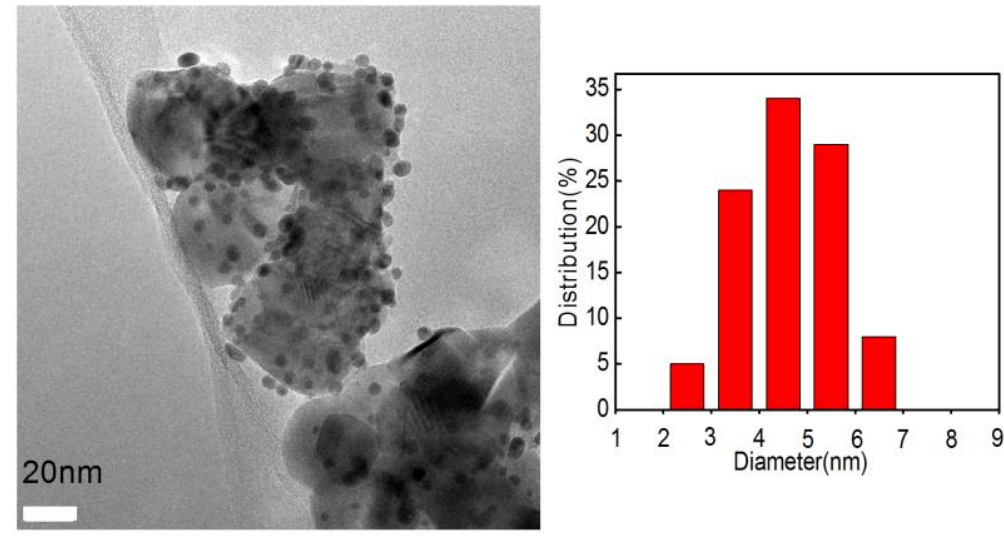

Figure 1. TEM image and the corresponding Au particles distribution of $3 \mathrm{wt} \% \mathrm{Au} / \mathrm{ZrO} 2$.

We also conduct XRD and XPS analysis to obtain further insights into the catalyst. Comparison of the powder XRD patterns of the pure $\mathrm{ZrO}_{2}$ and $\mathrm{Au} / \mathrm{ZrO}_{2}$ was provided in Figure 2. The diffraction peaks $\left(2 \theta=28.261^{\circ}, 31.562^{\circ}, 34.397^{\circ}, 50.160^{\circ}\right.$, etc. JCPDS data file $\left.37-1484\right)$ are assigned to the diffraction for the baddeleyite $\mathrm{ZrO}_{2}$ phase. No crystal Au can be detected by XRD, indicating that $\mathrm{Au}$ species is highly dispersed and the gold crystals are very small. 


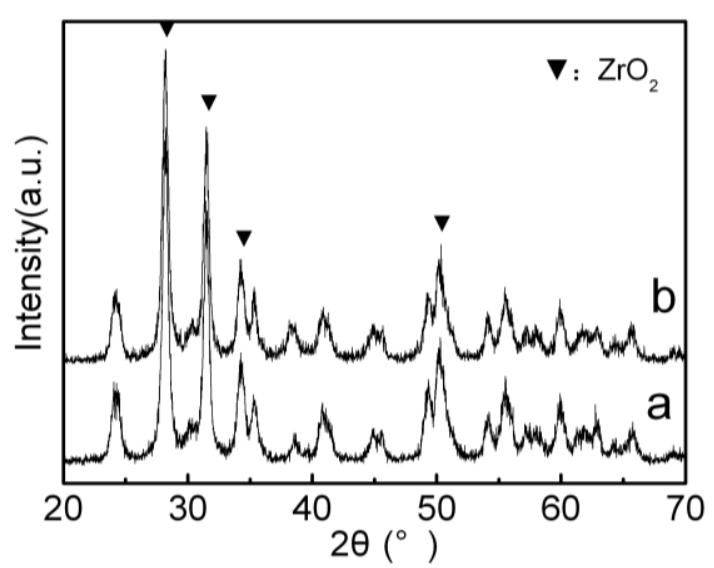

Figure 2. XRD pattern of (a) $\mathrm{ZrO} 2$ and (b) $3 \mathrm{wt} \% \mathrm{Au} / \mathrm{ZrO} 2$ catalyst.

X-ray photoelectron spectroscopy (XPS) was then performed with the objective to study the eventual state of $\mathrm{Au}$ on the surface of $3 \mathrm{wt} \% \mathrm{Au} / \mathrm{ZrO}_{2}$ nanoparticles. The $\mathrm{Au} 4 \mathrm{f} \mathrm{X}$-ray photoelectron spectroscopy (Figure 3) at $84.0 \mathrm{eV}$ and $87.65 \mathrm{eV}$ could be attributed to metallic $\mathrm{Au}$ 4f $7 / 2$ and $\mathrm{Au} 4 \mathrm{f}$ $5 / 2$, respectively. The most intense peak of the Au4f doublet (Au4f7/2) at a binding energy of $84.0 \mathrm{eV}$ is consistent with the presence of metallic Au particles.

\subsection{Activity results.}

The results of selective oxidation of benzyl alcohol to benzaldehyde on $\mathrm{Au} / \mathrm{ZrO}_{2}$ catalyst is presented in figure 4 . The catalyst of $3 \mathrm{wt} \% \mathrm{Au} / \mathrm{ZrO}_{2}$ exhibited high activity in the oxidation of benzyl alcohol as well as high selectivity to benzaldehyde at $40{ }^{\circ} \mathrm{C}$. Only in the presence of the supports or without any catalyst added, the yield of benzylaldehyde was below $2 \%$.

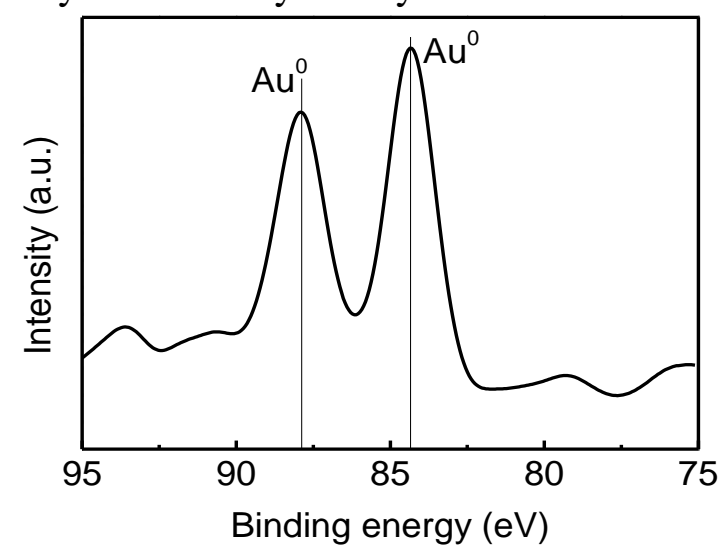

Figure 3. Au 4f XPS of 3 wt\% Au/ZO2 catalyst.

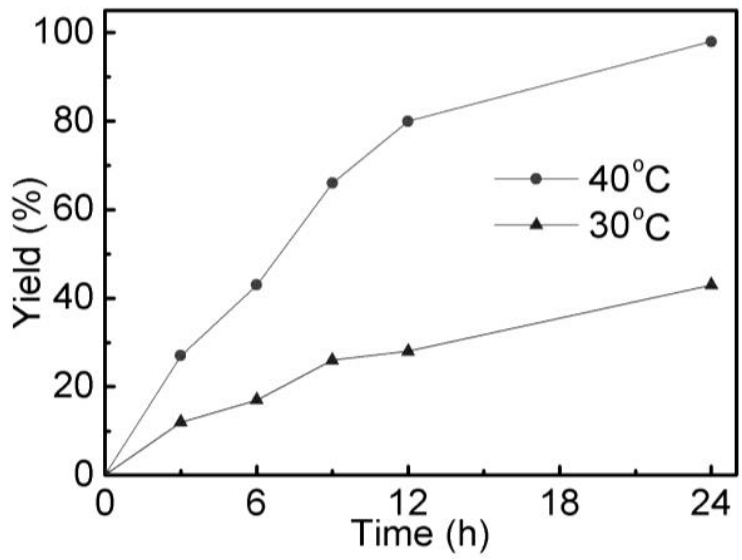

Figure 4. The effect of reaction temperature on the yield of benzaldehyde at different time. 


\section{Conclusion}

In summary, nano-gold catalyst was synthesized by solution reduction method, followed by characterization of the catalyst. On the basis of TEM and XRD results, $\mathrm{Au} / \mathrm{ZrO}_{2}$ catalyst with mean dimensions of about $4.5 \mathrm{~nm}$ exhibits high dispersion. According to XPS analysis, it can be interpreted as the Au nanoparticles are metallic state. The highly dispersed $\mathrm{Au} / \mathrm{ZrO}_{2}$ synthesized by this method showed significant activity toward benzyl alcohol oxidation even at $40{ }^{\circ} \mathrm{C}$.

\section{References}

[1]. Y. Zhang, X. Cui, F. Shi, Y. Deng, Chem. Rev. 112 (2012) 2467-2505.

[2]. G.J. Hutchings, Chem. Commun. 44 (2008) 1148-1164.

[3]. L. Aschwanden, T. Mallat, M. Maciejewski, ChemCatChem. 2 (2010) 666-673.

[4]. C.D. Pina, E. Falletta, M. Rossi, Chem. Soc. Rev. 41 (2012) 350-369. 